CrossMark \& click for updates

Cite this: DOI: $10.1039 / \mathrm{c5tc03529e}$

\title{
Structural and magnetic characterization of the double perovskite $\mathrm{Pb}_{2} \mathrm{FeMoO}_{6}^{\dagger}$
}

\author{
F. Mezzadri, ${ }^{a}$ D. Delmonte, ${ }^{b}$ F. Orlandi, ${ }^{a}$ C. Pernechele, ${ }^{c}$ G. Calestani, ${ }^{a}$ M. Solzi, ${ }^{c}$ \\ M. Lantieri, ${ }^{d}$ G. Spina, ${ }^{e}$ R. Cabassi, ${ }^{b}$ F. Bolzoni, ${ }^{b}$ M. Fittipaldi, ${ }^{e}$ M. Merlini, ${ }^{f}$ A. Migliori, ${ }^{g}$ \\ P. Manuel ${ }^{h}$ and E. Gilioli ${ }^{b}$
}

\begin{abstract}
The chemical and physical properties of the double perovskite $\mathrm{Pb}_{2} \mathrm{FeMoO}_{6}$ are systematically studied by means of structural and magnetic characterization. The compound crystallizes in the cubic Fm $\overline{3} m$ space group, with partial cation order involving iron and molybdenum at the perovskite B site. Structural and Mössbauer characterization points to the presence of nanometer-sized antiphase domains within the ordered matrix giving rise to two iron populations, characterized by different chemical environments, with the same weight but different valence ( 0.3 electrons) and inequivalent magnetic anisotropy. This structural feature deeply affects the properties of the compound: Mössbauer and EPR measurements show a high-temperature superparamagnetic-like behavior ascribed to weak magnetic interactions occurring between the antiphase domains and the rest of the sample. However, below $270 \mathrm{~K}$ ferrimagnetic ordering of the atomic moments is observed by neutron diffraction and SQUID magnetometry, with the onset of blocked long range magnetic interactions on the Mössbauer timescale involving both the antiphase domains and the ordered matrix below $230 \mathrm{~K}$. The superparamagnetic-like behavior is ascribed to the presence of low anisotropy barriers, giving rise to an extremely thin hysteresis loop at $5 \mathrm{~K}$, with a very small coercive field and remnant magnetization. The observed saturation magnetization of $1.75 \mu_{\mathrm{B}}$ per f.u. is in agreement with the magnetic structure determined by neutron diffraction, with the two symmetry independent sites producing a ferrimagnetic resultant $\mu_{\mathrm{S}}=1.59 \mu_{\mathrm{B}}$.
\end{abstract}

Received 28th October 2015, Accepted 19th January 2016

DOI: $10.1039 / \mathrm{c} 5 \mathrm{tc} 03529 \mathrm{e}$

www.rsc.org/MaterialsC a further degree of freedom represented by the splitting of the B site allows in principle the fine-tuning of cation ordering, magnetic exchange interactions and electrical properties which give rise to widespread exotic features. As a direct consequence, a large number of double perovskites are known to show peculiar properties, as for example multiferroicity, ${ }^{1}$ spin polarized carriers, ${ }^{2}$ magnetoresistance ${ }^{3}$ or catalytic properties. ${ }^{4}$ Compounds having the B and B' sites occupied by ions with different ionic radii and/or oxidation states are inclined to cation ordering, often showing half-metallic properties due to the coexistence of bound and delocalized electrons. Within this framework, $\mathrm{Sr}_{2} \mathrm{FeMoO}_{6}$ can be considered a prototypical system, widely studied in particular due to its high magnetic transition temperature $\left(T_{\mathrm{C}}=420 \mathrm{~K}\right)$ inducing room-temperature magnetoresistance, which makes the compound an interesting candidate for applications. ${ }^{3,5}$ The presence of both the $\mathrm{Fe}^{3+}\left(3 \mathrm{~d}^{5}\right)$ and $\mathrm{Mo}^{5+}\left(4 \mathrm{~d}^{1}\right)$ ions confers to the material half metallicity ${ }^{6}$ and ferromagnetic properties, ${ }^{7}$ strongly affected by the possible presence of antisite defects. ${ }^{8}$ The synthesis conditions are reported to play a central role in determining the degree of the cation order, ${ }^{7}$ and consequently the properties of the system, in particular the application of high pressure during the solid state reaction process is reported to decrease the amount of antisite defects. ${ }^{9}$ The substitution of 
$\mathrm{Sr}^{2+}$ by the isovalent ion $\mathrm{Pb}^{2+}$ is expected to induce ferroelectric properties to the system, due to its stereoactive $6 \mathrm{~s}^{2}$ lone pair, allowing in principle multiferroicity through the coexistence of electric and magnetic orders. Based on the first principles calculations, the compound is suggested to be half metallic, ${ }^{10,11}$ while experimental studies indicate that it orders magnetically at about $275 \mathrm{~K},{ }^{12}$ showing low-field magnetoresistance, ${ }^{13}$ ascribed to the field-induced suppression of spin fluctuations. The magnetic properties appear to be affected by the degree of cation order, likely influenced by the synthesis conditions, while a thermally activated electron conduction process is detected, ascribed to variable-range hopping of the carriers. ${ }^{13}$ However, at present detailed magnetic characterization of the compound is missing, while little is known about the crystal structure of $\mathrm{Pb}_{2} \mathrm{FeMoO}_{6}$. As a consequence we decided to perform complete crystallographic characterization in order to extend the knowledge of both the nuclear and magnetic structures of the compound by using electron diffraction (ED), synchrotron X-ray diffraction (SXRD) and neutron time of flight (TOF) measurements. Magnetometric, Mössbauer and electron paramagnetic resonance (EPR) characterization gives a deep insight into the magnetism of the system, finally allowing us to relate the structural and magnetic properties of the compound.

\section{Experimental}

\subsection{Synthesis}

The samples were obtained through a solid state reaction under high pressure/high temperature (HP/HT) conditions by using a multianvil apparatus. Stoichiometric amounts of $\mathrm{PbO}, \mathrm{FeO}$ and $\mathrm{MoO}_{3}$ were ground and encapsulated into a gold foil. The system was pressurized to $5 \mathrm{GPa}$ and then heated to $900{ }^{\circ} \mathrm{C}$ for $1.5 \mathrm{~h}$ of reaction time. After quenching the temperature, pressure was slowly released.

\subsection{Structural characterization}

Electron diffraction experiments were carried out by using a Philips TECNAI F20 transmission electron microscope (TEM) operating at $200 \mathrm{kV}$. The samples were ground, suspended in isopropyl alcohol, and then a few drops of the solution were evaporated on a copper grid coated with an amorphous carbon film.

Synchrotron X-ray diffraction data were collected at the ID09A beamline at the ESRF in Grenoble, France. The powdered sample was introduced into a $0.2 \mathrm{~mm}$ boron-glass capillary and data were collected in transmission using a flat panel MAR555 detector. The data were integrated using the Fit2D software, ${ }^{14}$ taking into account the polarization and geometrical correction. The X-ray wavelength was set to $0.41464 \AA$.

Time of Flight (TOF) neutron diffraction experiments were carried out on the WISH instrument ${ }^{15}$ at the ISIS facility, Didcot, UK. The diffraction data were collected in the temperature range of $1.5-310 \mathrm{~K}$ on $265 \mathrm{mg}$ of the powdered sample, using thin vanadium can as a sample holder placed in a standard cryostat with exchange gas.
The nuclear and magnetic structure refinement was carried out using the GSAS package ${ }^{16}$ with the EXPGUI interface, ${ }^{17}$ by using the data collected on the WISH banks with $2 \theta=152.8^{\circ}$, $121.6^{\circ}, 90.0^{\circ}$ and $58.3^{\circ}$. Magnetic symmetry analysis was performed with the help of the ISODISTORT software. ${ }^{18}$

\subsection{Magnetometry}

Magnetic measurements were performed by using a Superconducting Quantum Interference Device (SQUID) magnetometer MPMS-XL. Fragments of the sintered bulk samples obtained via HP/HT synthesis were used for the collection of $\mathrm{M}(\mathrm{T})$ and $\mathrm{M}(\mathrm{H})$ curves in the standard DC mode. For the magnetic susceptibility $v s$. temperature measurements the procedures of Zero Field Cooled (ZFC) and Field Cooled (FC) were performed, under warming and cooling conditions, respectively. Magnetic measurements above room temperature (RT) were performed by using a DSM8 stationary pendulum magnetometer equipped with an oven operating in the temperature range of $\mathrm{RT}-850{ }^{\circ} \mathrm{C}$.

\subsection{Mössbauer and EPR}

Mössbauer spectra were collected by means of a $\mathrm{Kr}-\mathrm{CO}_{2}$ proportional counter, Fast ${ }^{\mathrm{TM}}$ electronics for gamma ray spectroscopy and a Wissel $^{\mathrm{TM}}$ spectrometer, which was run in the sinusoidal acceleration mode $\left(v_{\max }=12 \mathrm{~mm} \mathrm{~s}^{-1}\right)$ and calibrated by using a standard metal iron foil. Twenty measurements were carried out between $67 \mathrm{~K}$ and $305 \mathrm{~K}$ by using a nitrogen-based Oxford ${ }^{\mathrm{TM}}$ flux cryogenic system with a base temperature of $67 \mathrm{~K}$ at 232 mbar. The $\gamma$-ray source was $25-\mathrm{mCi}{ }^{57} \mathrm{Co}$ in a rhodium matrix with Lamb-Mössbauer factor $f_{\mathrm{s}}=0.615$, as measured by applying the method described in ref. 19. Since we were interested in evaluating the magnetic components of the spectra, we needed a sample with a high value of the total effective thickness $t_{\mathrm{a}}$. Therefore, the compound for Mössbauer analysis was synthesized using $48 \%$ of ${ }^{57} \mathrm{Fe}$ isotope. The Mössbauer sample contained $18.2 \mathrm{mg} \mathrm{cm} \mathrm{cm}^{-2}$ of compound, corresponding to an expected $t_{\mathrm{a}}$ value of $\approx 20 f_{\mathrm{a}}$, where $f_{\mathrm{a}}$ is the absorber recoilless fraction.

X-band EPR measurements were performed using a $9 \mathrm{GHz}$ Bruker Elexys E500 instrument equipped with a microwave frequency counter. An Oxford Instruments ESR 900 continuous He flow cryostat was used to obtain low temperatures. EPR spectra were acquired using a field modulation of $100 \mathrm{kHz}$ and $9 \mathrm{G}$, and a microwave power of $5 \times 10^{-4} \mathrm{~mW}$.

\section{Results}

\subsection{Crystal structure}

TEM experiments were performed in order to evaluate the structural properties of $\mathrm{Pb}_{2} \mathrm{FeMoO}_{6}$ at the nanometre scale. Selected area electron diffraction (SAED) patterns collected in different zone axes show the presence of superstructure spots requiring the doubling of the simple perovskite lattice parameters in all the directions (Fig. 1). All the observed reflections can be indexed in a cubic cell with lattice parameter $a^{\prime}=2 a_{\mathrm{p}}$, 


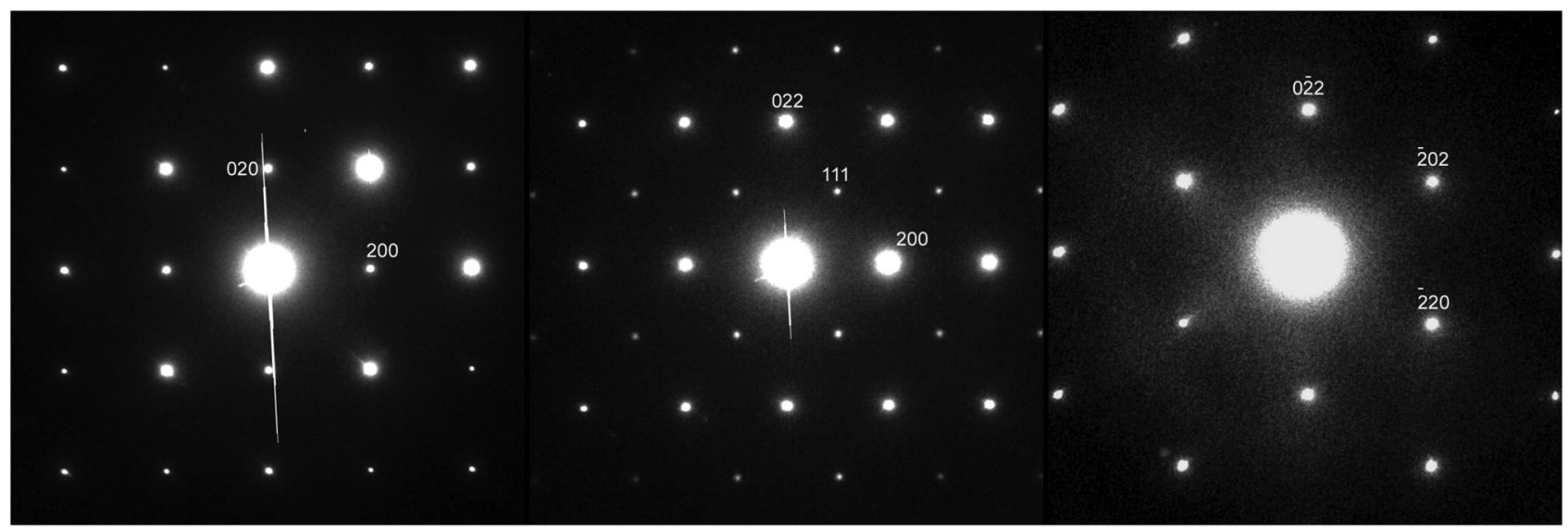

Fig. 1 SAED patterns taken in the [001], [01-1], and [111] (left to right) zone axes. The reflections are indexed on the basis of the supercell with $a^{\prime}=2 a_{\mathrm{p}}$.

where $a_{\mathrm{p}}$ indicates the simple perovskite, and the systematic absence of the $h+k, k+l, h+l=2 n+1$ reflections indicates F-centering of the cell. The simultaneous use of SXRD and TOF neutron data allowed the accurate refinement of the room temperature (RT) crystal structure of $\mathrm{Pb}_{2} \mathrm{FeMoO}_{6}$. No deviation from the cubic symmetry was detected and, according to electron diffraction, Rietveld refinements were performed in all the space groups having the same extinction rules: $F m \overline{3} m$, $F \overline{4} 3 m, F 432, F 4_{1} 32, F m \overline{3}$, and $F 23$. $F m \overline{3} m$ gave the most reliable result allowing the highest symmetry, thus not requiring an increase of the free parameters, as a consequence it was selected as the correct one. The refined cell parameter is $a=7.9609(4) \AA$; the F-centering, together with the presence of weak satellite reflections with odd indices detected in both the synchrotron and neutron data, suggests the presence of cation ordering. As a consequence, the occupation of the B sites, initially set at a $\mathrm{Fe} / \mathrm{Mo}$ ratio equal to 1 , was freely refined in order to evaluate the distribution of the different atomic species at the two independent crystallographic sites. Two additional phases, $\mathrm{PbMoO}_{4}$ and $\mathrm{Au}$, were added in the refinement in order to account for the extra peaks detected. The refined weight ratios are $6.32 \%$ and $2.97 \%$ for the two spurious phases. The refinement converged with agreement factors $\mathrm{w} R_{\mathrm{p}}=4.84 \%, R_{\mathrm{p}}=3.59 \%$, averaged over the four neutron and one synchrotron diffraction histograms. The individual agreement factors of the histograms are reported together with the corresponding Rietveld plots in Fig. 2 (synchrotron data) and Fig. S1-S4 in the ESI $\dagger$ (TOF patterns), where also a table gathering all the refined parameters and crystallographic data can be found.

Rock salt-like cation ordering is detected, both the B sites being characterized by the presence of antisite defects or antiphase domains with dimensions below the coherence length of both X-rays and neutrons, thus involving the presence of iron at the molybdenum site and vice versa. The antisite defects are distributed in ratios of $17 \%$ and $27 \%$ on the two sites, yielding an average value of $22 \%$. Hereinafter the two sites will be indicated as Fe1 and Mo1, corresponding to the species with the major occupancy within each site. The attempt to refine both the lead and oxygen occupancies did not yield relevant

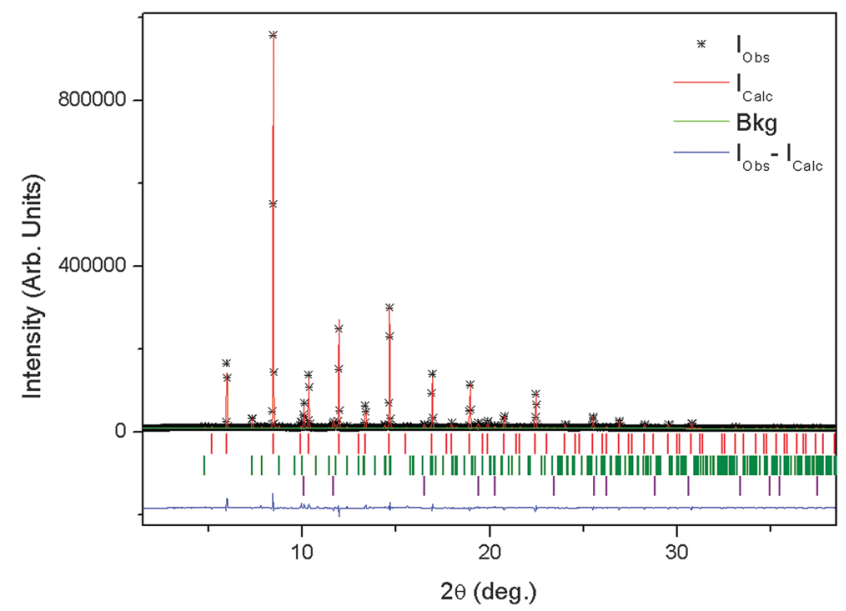

Fig. 2 Rietveld plot of SXRD data collected with $\lambda=0.41464 \AA$. The symbols indicate the experimental data, the red line the computed pattern while the blue line at the bottom the experimental-calculated intensity difference. Thick marks (top to bottom): $\mathrm{Pb}_{2} \mathrm{FeMoO}_{6}$ (pink), $\mathrm{PbMoO}_{4}$ (green), and $\mathrm{Au}$ (purple).

deviation from the starting value of 1 , as a consequence the two sites were considered to be fully occupied. The obtained information points to a slight iron excess, yielding the composition $\mathrm{Pb}_{2} \mathrm{Fe}_{1.1} \mathrm{Mo}_{0.9} \mathrm{O}_{6}$, which can be expected in the presence of $\mathrm{PbMoO}_{4}$ as a spurious phase. By considering the refined amounts of the two phases a $\mathrm{Pb}: \mathrm{Fe}:$ Mo ratio in agreement with the nominal composition is obtained. It should be noticed that the $F m \overline{3} m$ space group does not allow any tilt or rotation of the $\mathrm{BO}_{6}$ coordination octahedra nor polyhedral distortions (as for example Jahn-Teller effect or ion off-centering); however different B-O bond distances are permitted, according to the presence of (partial) cation ordering. The refined Fe1-O bond length is, indeed, 1.995(3) A, while Mo1-O is 1.987(3) A, suggesting, on the basis of bond valence sum (BVS) calculations, the presence of $\mathrm{Fe}^{3+}$ and $\mathrm{Mo}^{5+}$ within the structure. ${ }^{20}$ It is noteworthy that a local distortion of the $\mathrm{Pb}$ environment is detected. Indeed, when refinements are carried out with the lead atom fixed at the high symmetry $(1 / 4,1 / 4,1 / 4)$ site, 
Table 1 Selected bond lengths

\begin{tabular}{ll}
\hline Atoms & Distance $(\AA)$ \\
\hline Fe1-O1 $(\times 6)$ & $1.995(3)$ \\
Mo1-O1 $(\times 6)$ & $1.987(3)$ \\
Pb1-O1 $(\times 3)$ & $2.594(2)$ \\
Pb1-O1 $(\times 6)$ & $2.830(3)$ \\
Pb1-O1 $(\times 3)$ & $3.048(2)$
\end{tabular}

anomalously large isotropic displacement parameters, suggesting the static disorder, are observed.

In contrast a small shift of the $\mathrm{Pb}$ atoms, disorderly distributed along the threefold axes, leads to a normalization of the thermal parameters. The list of the relevant bond distances is reported in Table 1. The statistical displacement from the high symmetry position gives rise to $\mathrm{Pb}-\mathrm{O}$ bond lengths in the 2.594-3.048 $\AA$ range, in line with the typical stereochemistry of the $\mathrm{Pb}^{2+}$ ion. A representation of the crystal structure of $\mathrm{Pb}_{2} \mathrm{FeMoO}_{6}$ is shown in Fig. 3. The refinement of TOF data collected below room temperature allowed the exhaustive study of the evolution of both the nuclear and magnetic structures of $\mathrm{Pb}_{2} \mathrm{FeMoO}_{6}$. No structural phase transition is observed, the cubic symmetry persisting in the whole explored range (1.5-300 K). Just below RT the intensity of the (111) reflection increases sharply, suggesting the development of a magnetic structure with propagation vector $\kappa=(0,0,0)$. The neutron diffraction patterns collected at RT and $1.5 \mathrm{~K}$ are compared and reported in Fig. S5 (ESI $\dagger$ ). Since in the $F m \overline{3} m$ space group (and in the other cubic space groups) the site symmetry of the $4 \mathrm{a}$ and $4 \mathrm{~b}$ positions, occupied in the present case by the magnetic ions, does not allow a spontaneous magnetic moment, a symmetry lowering is necessary in order to fit the magnetic signal. This is in agreement with the phenomenology of $\mathrm{Sr}_{2} \mathrm{FeMoO}_{6}{ }^{7}$ where the onset of long-range magnetic interactions

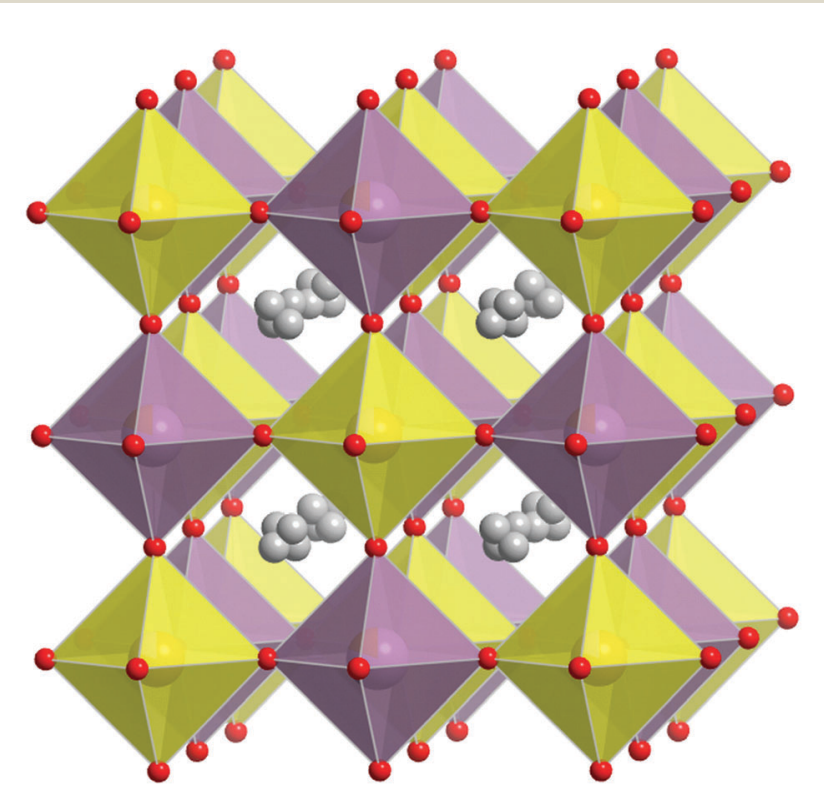

Fig. $3 \mathrm{~Pb}_{2} \mathrm{FeMoO}_{6}$ unit cell viewed along one of the [100] directions Green and purple octahedra are occupied by molybdenum and iron, respectively; grey spheres represent the lead atoms statistically disordered around the $8 \mathrm{c}$ position; the oxygen ions are drawn in red. ${ }^{21}$ induces a cubic to tetragonal transition which however is reported to involve also the nuclear structure.

Similarly, within instrumental resolution no transition involving the nuclear structure is detected, so that just the magnetic structure was refined in a lower symmetry space group. Several subgroups of the $F m \overline{3} m$ space group were considered, namely $R \overline{3} m^{\prime}, I 4 / \mathrm{mm}^{\prime} m^{\prime}$, $I m^{\prime} m^{\prime} m$ and $C 2^{\prime} / m^{\prime}$, all corresponding to the mGM4+ representation with different directions of the order parameter, giving identical results in terms of the relative orientation of the spins. The resultant magnetization direction within the fundamental cubic reference is prevented. Therefore, owing to the absence of a detectable lattice distortion, the univocal determination of the magnetization measurements performed on single crystals would be required to determine the easy axis of magnetization of the system, and univocally determine the magnetic space group.

For instance, in the case of the high symmetry rhombohedral and tetragonal subgroups, the resultant magnetization would lie along the [111] and [001] directions of the parent cubic cell, respectively. The coloured magnetic space group $I 4 / \mathrm{mm}^{\prime} \mathrm{m}^{\prime}$ was used for the refinements, involving time inversion symmetry operating on the mirror planes perpendicular to the [100] and [110] directions, in analogy with $\mathrm{Sr}_{2} \mathrm{FeMoO}_{6}$. The Fe1 and Mo1 site moments were refined independently, converging to an antiparallel arrangement characterized by magnetizations of 3.06(3) $\mu_{\mathrm{B}}$ and $-1.47(4) \mu_{\mathrm{B}}$, respectively. A sketch of the magnetic structure is reported in Fig. 4. The thermal evolution of the moments is reported in Fig. 5, showing different behaviours for the two transition metal (TM) sublattices. On the one hand indeed, the Fe1 moment develops smoothly, reaching saturation at about $60 \mathrm{~K}$; on the other hand, the one of Mo1 stabilizes at $250 \mathrm{~K}$, just after an initial sharp increase probably produced by the magnetization of the surrounding iron atoms. The evolution of the ferrimagnetic resultant moment can be calculated as $\mu(\mathrm{Fe} 1)-\mu(\mathrm{Mo1})$ for different temperatures.

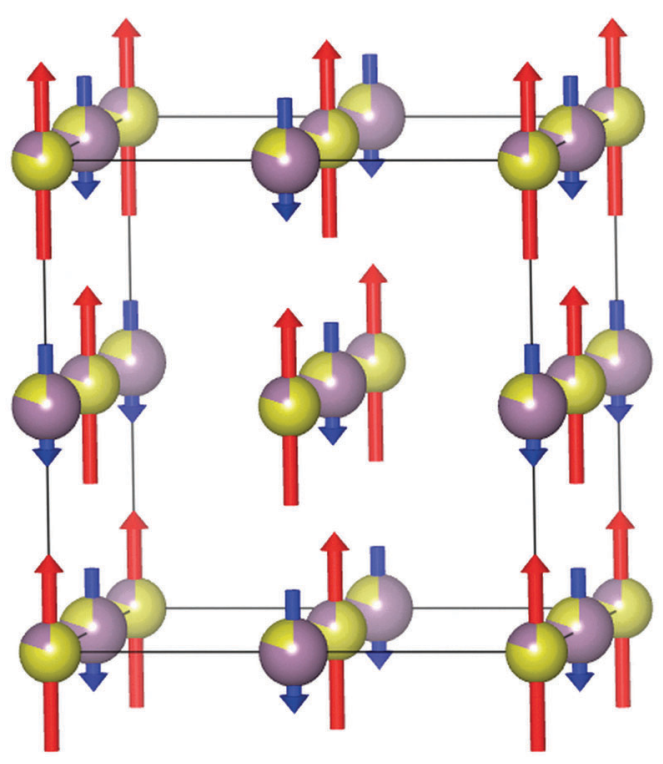

Fig. 4 Magnetic structure of $\mathrm{Pb}_{2} \mathrm{FeMoO}_{6}$. In red and blue the atomic moments of iron and molybdenum are represented, respectively. 


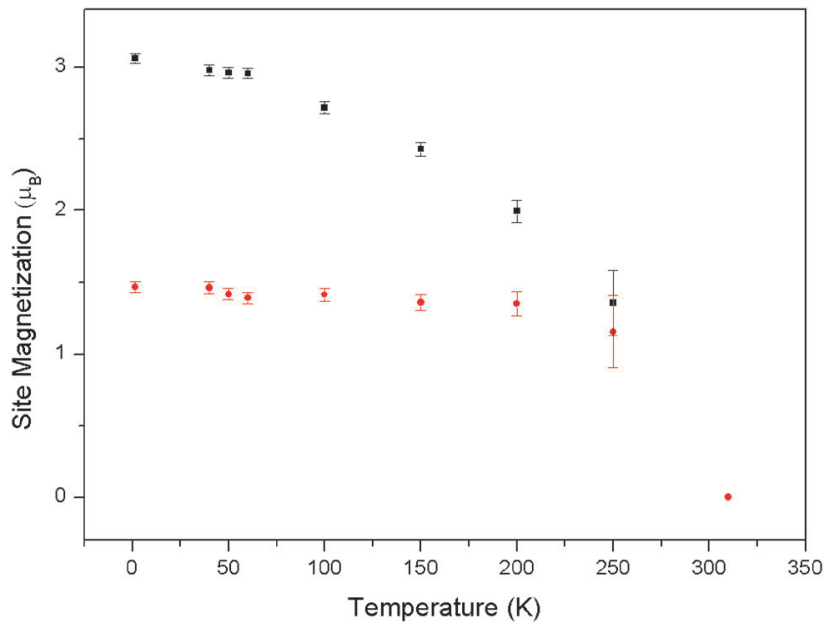

Fig. 5 Site magnetization evolution as a function of temperature as obtained by the refinement of neutron diffraction data. In black and red the site moments of Fe1 and Mo1 are represented, respectively.

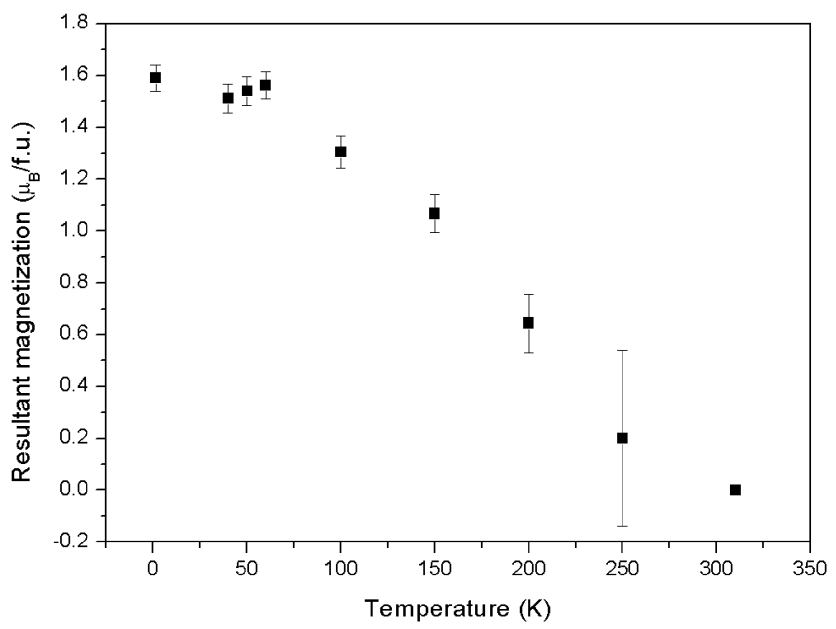

Fig. 6 Saturation magnetization per formula unit as obtained by the refining of the neutron diffraction data as $\mu(\mathrm{Fe} 1)-\mu(\mathrm{Mo} 1)$.

The plot, shown in Fig. 6, indicates a $T_{\mathrm{N}}$ of about $270 \mathrm{~K}$, a smooth increase of magnetic moment followed by a stabilization with a slight loss of resultant magnetization below $60 \mathrm{~K}$. Based on these data the expected saturation moment is $1.59 \mu_{\mathrm{B}}$.

\subsection{Magnetic characterization}

The magnetic characterization of $\mathrm{Pb}_{2} \mathrm{FeMoO}_{6}$ was focused on the study of its intrinsic properties through an accurate analysis of the magnetization dependence on both the temperature and the applied field. Spontaneous magnetization was recorded versus temperature using a combined ZFC-FC procedure applying an external field of 100 Oe (Fig. 7). As it will be shown in the following, the mean negative sign of the Weiss constant indicates that the nature of the transition observed at $T=272 \mathrm{~K}$ is ferrimagnetic. A nonzero magnetization indeed is developed, indicating a clear long range magnetic order arising from a paramagnetic disordered state. Nevertheless at lower temperature

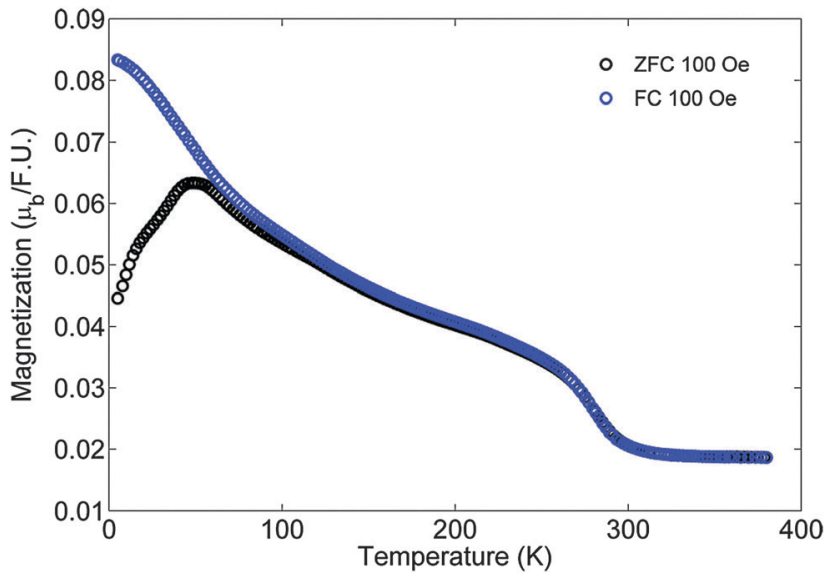

Fig. 7 ZFC FC susceptibility of a polycrystalline pellet of $\mathrm{Pb}_{2} \mathrm{FeMoO}_{6}$, performed at $100 \mathrm{Oe}$ in the range of $5-380 \mathrm{~K}$.

the ZFC curve highlights a second phenomenon that brings to a maximum at about $55 \mathrm{~K}$ and then to a decrease of the susceptibility as the system is cooled down to $5 \mathrm{~K}$. This behaviour may be ascribed to the blocking temperature of a superparamagnetic phase enclosed into the ferrimagnetic matrix, corresponding to the one seen near $230 \mathrm{~K}$, with different measuring times, using Mössbauer and EPR techniques (see the following sections). ZFC-FC measurements performed in the presence of a high applied field (1 kOe), reported in Fig. 8, show similar behaviour with respect to the 100 Oe case, with the same characteristic temperatures and ferrimagnetic contribution. In order to coherently interpret and describe the global nature of the interactions, $M(T)$ measurements have been performed at higher temperatures, reaching the thermal region in which the paramagnetic state engages all the volume of the material. This operation has been successfully carried out at $10 \mathrm{kOe}$ and above $400 \mathrm{~K}$, where the system effectively shows a complete paramagnetic behaviour.

The need of such a high field is due to the weak sensitivity of the used technique (the stationary pendulum magnetometer) together with the small mass of the tested sample. In this way,

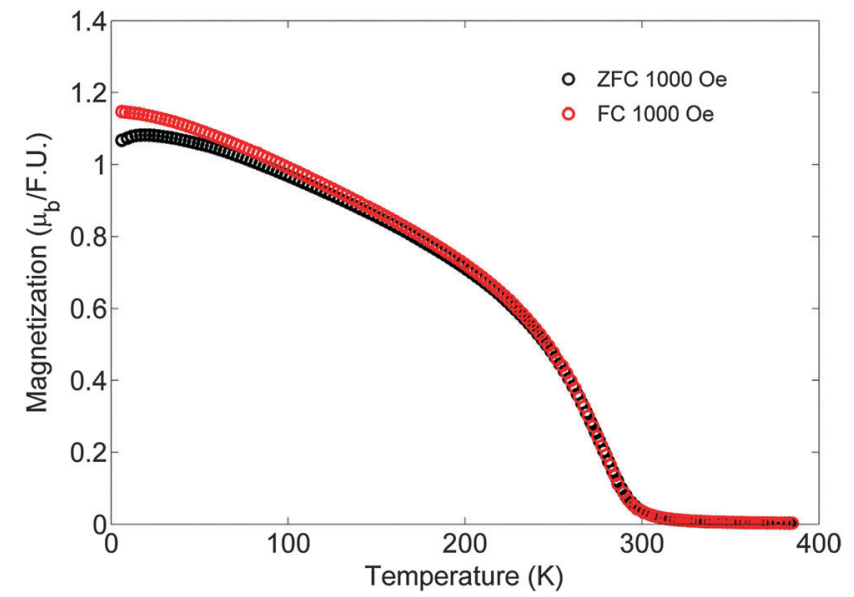

Fig. 8 ZFC FC susceptibility measured at 1000 Oe in the range of 5-380 K. 


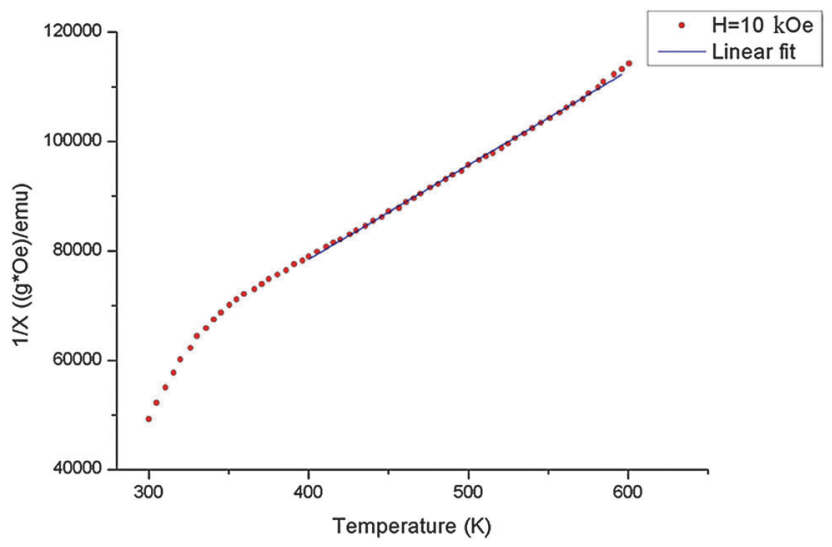

Fig. 9 Curie Weiss fit in the paramagnetic regime of $\mathrm{Pb}_{2} \mathrm{FeMoO}_{6}$ of the inverse magnetic susceptibility collected at $10 \mathrm{kOe}$.

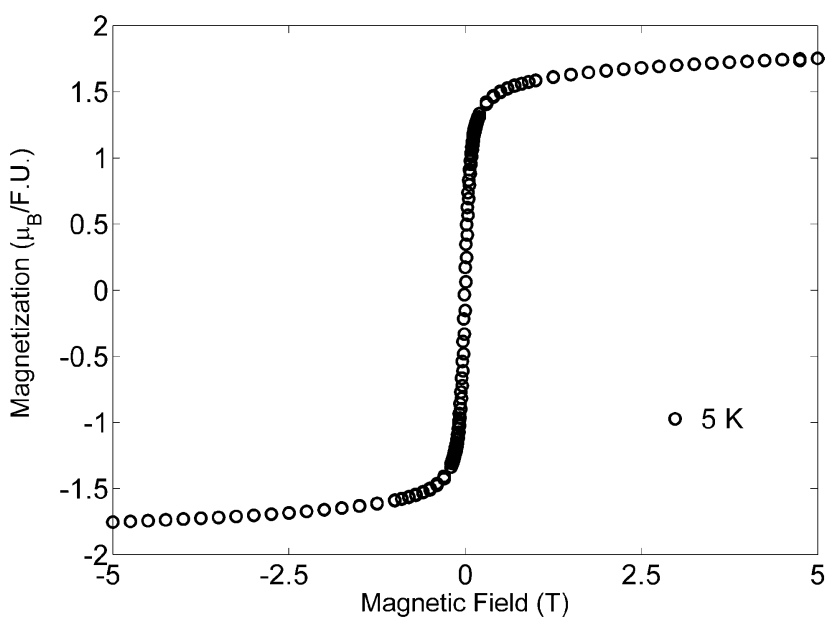

Fig. 10 Magnetization vs. applied field measurement collected at $5 \mathrm{~K}$

by means of a linear fit of the inverse magnetic susceptibility (Fig. 9), we were able to obtain the experimental values of $\Theta=-(55 \pm 6) \mathrm{K}$ and $(5.55 \pm 0.25) \mu_{\mathrm{B}}$ per f.u., with good statistics. The negative sign of $\Theta$, related to the mean sign of the exchange integral, is compatible with an antiferromagnetic and/or ferrimagnetic system; on the other hand the shape of the curve, characterized by the onset of nonzero magnetization, is typical of a ferrimagnetic system. The curve deviates from its linear trend below $350 \mathrm{~K}$, indicating the onset of magnetic interactions above RT, in agreement with the Mössbauer data reported in the following section. The presence of a negative Weiss constant together with the clear onset of nonzero magnetization indicates the system as a ferrimagnet characterized by the prevalent superexchange interaction $\mathrm{Fe}^{3+}-\mathrm{O}-\mathrm{Mo}^{5+}$. A study of the $5 \mathrm{~K}$ magnetization as a function of the applied magnetic field was performed spanning the range $-5 \mathrm{~T}$ to $5 \mathrm{~T}$ by performing a double cycle, and the results are plotted in Fig. 10.

The measurement presents the H-dependent trend of a magnetic ordered system with a spontaneous magnetic moment per unit cell $M_{\mathrm{S}}=1.75 \mu_{\mathrm{B}}$ per f.u. obtained symmetrically at $-5 \mathrm{~T}$ and $5 \mathrm{~T}$. The presence of such saturation confirms that the system behaves as a ferrimagnetic material and, in parallel, it is incompatible with an antiferromagnetic response.

\subsection{Mössbauer}

The spectra collected at around RT (see Fig. 11 and Fig. S7, ESI $\dagger$ ) are characterized by a deep and asymmetrical single line overlapped to two magnetically ordered components, identified by different isomer shifts and hyperfine fields of $\approx 45 \mathrm{~T}$ and $\approx 50 \mathrm{~T}$, respectively and null quadrupole splittings, as expected on the basis of the crystallographic data previously reported. The isomer shift difference determines the superposition of the sixth lines of the two sextets. By lowering $T$, the single line component exponentially decreases in favor of magnetically ordered contributions, revealing a thermal evolution typical of superparamagnetic nanoclusters. ${ }^{22,23}$ Moreover, the collapse of the asymmetrical central line leads to a magnetic structure which is characterized by an asymmetry similar to the one of the RT magnetically ordered component. So, the spectra are surely due to contributions belonging to different kinds of nanoclusters, characterized by different isomer shifts and by a correlation between the hyperfine field and isomer shift values. Moreover, the asymmetry of the RT central line indicates an additional correlation between the isomer shift and the anisotropy barrier or the percentage amount of nanoclusters. A phenomenological interpretation of the temperature dependence of spectra due to magnetic nanoclusters requires the use of a set of sub-spectra: the high-energy states, lying over the anisotropy barrier, give rise to the superparamagnetic-like single line and the low-energy states, located inside the barrier, give rise to the remaining contribution to the Mössbauer cross-section $\sigma_{\mathrm{M}}(E)$. The latter contribution is characterized by a T-dependent distribution of hyperfine fields and can be approximated by a sum of Voigt sextets. Consequently, the standard procedure applied to describe hyperfine field distribution ${ }^{24}$ and the scheme adopted in ref. 25 can be used in the present case. In order to limit the total number of base functions, we introduced only two kinds of clusters and four sub-spectra for each of them, obtaining eight base sub-spectra in total. Furthermore, just to take the anisotropy

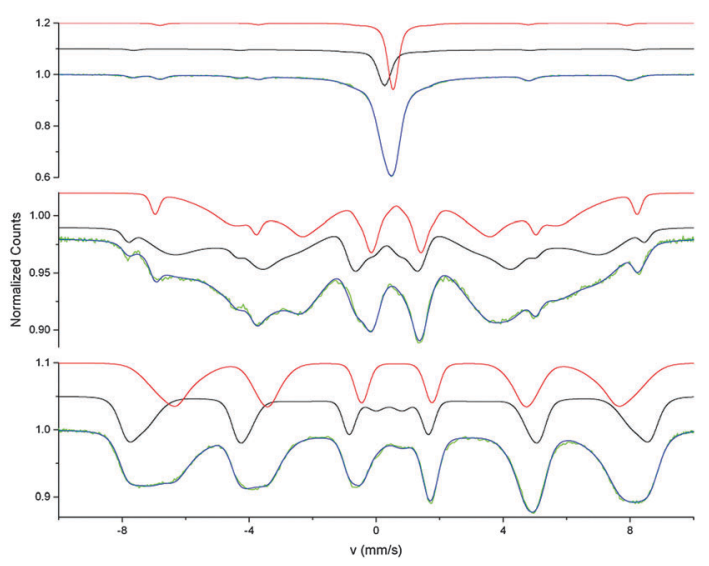

Fig. 11 Mössbauer spectra (blue line) collected at $67 \mathrm{~K}, 195 \mathrm{~K}$ and RT (bottom to top) with corresponding fit curves (red and black lines) and site cross-sections. 
broadenings into account, the Gaussian component of the single line, which belongs to each Voigt sextet, is given by a suitable quadratic sum of standard deviations of the electric and the magnetic parameter values. Preliminary fits, performed using the procedure detailed in the following but without any assumption regarding the percentage contributions to the sample thickness, show that the Mössbauer line shape can be reproduced by two magnetic components of almost equal weight in the whole temperature range.

In particular, the high temperature spectra suggest that the percentage weight of the two clusters differs from each other by less than $10 \%$. The estimated difference progressively decreases by lowering $T$ and becomes lower than $1 \%$ at $67 \mathrm{~K}$. Consequently, $\sigma_{\mathrm{M}}(E)$ was expressed by the sum of two components with the same weight, due to the two kinds of clusters characterized by two different potential wells.

- the quadrupole splitting values are set to zero, as suggested by the ordered component of the RT spectrum;

- the weight of the two components of $\sigma_{\mathrm{M}}(E)$ is the same;

- the inhomogeneous broadenings related to the electrical hyperfine parameters are the same for the homologous sextets belonging to the two different components. The most relevant information obtained from the fit of the spectra is derived from the temperature dependencies of the isomer shifts, the hyperfine field densities, and the total thickness number of the sample and of the superparamagnetic-like state contribution to $t_{\mathrm{a}}$. The isomer shifts for both the components follow identical thermal trends, which can be reproduced on the basis of the Debye model and in the whole temperature range they deviate from each other for $0.25 \mathrm{~mm} \mathrm{~s}^{-1}$ (Fig. S8, ESI $\dagger$ ), corresponding to a difference of $\approx 0.3$ electrons in the iron 3d-orbital occupation.

This result is also confirmed by $B_{\mathrm{hf}}$ values for the two magnetically ordered components at RT, which deviate from each other for 3.5 T, assuming that a Fe(III) ion with spin $s=5 / 2$ gives rise to $B_{\mathrm{hf}}=55 \mathrm{~T}$. The total effective thickness $t_{\mathrm{a}}$ follows a thermal trend well described by a Debye model with $\theta_{\mathrm{D}}=318 \mathrm{~K}$, as shown in Fig. S9 (ESI $\dagger$ ). Moreover, the superparamagneticlike components for both the sub-spectra fall to zero for $T \approx 225 \mathrm{~K}$ and show a quasi-flat trend between $250 \mathrm{~K}$ and $280 \mathrm{~K}$, as illustrated in Fig. 12. As a consequence of the collapse of the superparamagnetic-like contributions to the spectra, the magnetic hyperfine field densities change from broadened to sharpened distributions characterized by mean values close to the ones obtained at room temperature for the ordered components of the spectrum (Fig. S10, ESI $\dagger$ ). Starting from the hyperfine field distributions and assuming that a Fe(III) ion with spin $s=5 / 2$ gives rise to $B_{\mathrm{hf}}=55 \mathrm{~T}$, the Fe ion magnetic moment was estimated, obtaining the thermal trend illustrated in Fig. 13, where in particular, $\mu_{\mathrm{Fe}}(T=67 \mathrm{~K}) \approx 3.95 \mu_{\mathrm{B}}$ and $\mu_{\mathrm{Fe}}(T=305 \mathrm{~K}) \approx 0.87 \mu_{\mathrm{B}}$. The trend obtained by Mössbauer spectroscopy is compared in Fig. 13 with the one refined for the Fe1 site by neutron diffraction. Since the Fe1 site contains both iron and molybdenum, the curve was shifted in order to compare, from the qualitative point of view, the magnetization evolution in the two cases, revealing excellent agreement at low temperatures, while a slight difference is observed at above $200 \mathrm{~K}$,

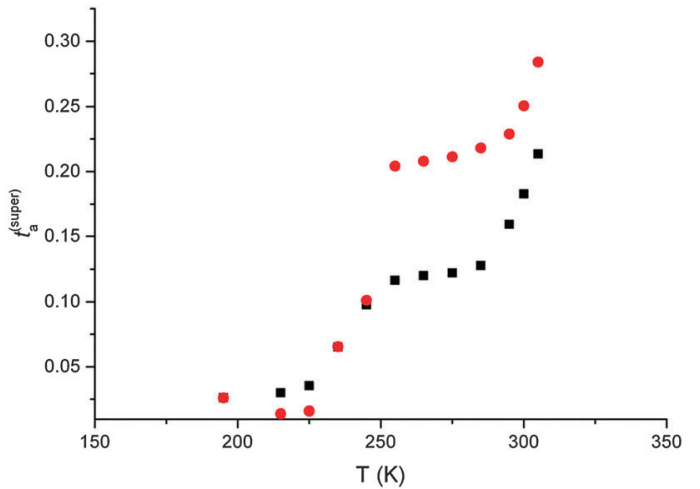

Fig. 12 Normalized superparamagnetic-like contributions to ta(1) (red) and ta(2) (black).

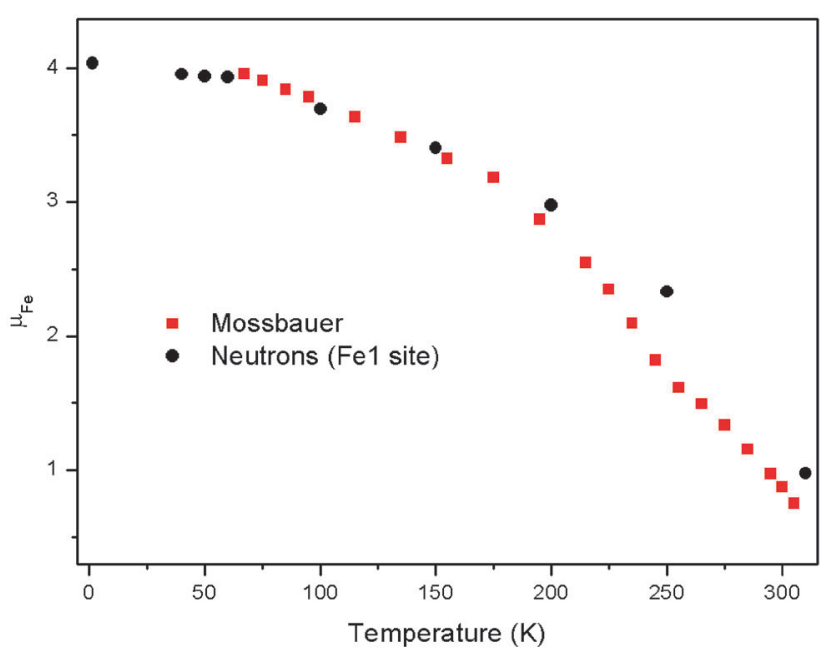

Fig. 13 Magnetic moment for iron ions vs. $T$ as determined by Mössbauer (red squares) and neutron diffraction (black dots). The latter data were translated to subtract the contribution of molybdenum.

probably pointing out the role of molybdenum in the magnetization evolution of the Fe1 site.

\subsection{Electron paramagnetic resonance}

EPR spectra, recorded at variable temperature, are illustrated in Fig. 14. The main feature of the thermal evolution of the spectra is a broadening of the resonance line upon lowering the temperature and a gradual shift towards lower magnetic field values. In order to describe this behaviour more accurately, the temperature behaviour of the EPR intensity and the line width are reported in Fig. 15.

Between RT and $280 \mathrm{~K}$ an increase in the EPR line width (Fig. 15, right side) and a decrease in the normalized contributions to $t_{\mathrm{a}}(1)$ and $t_{\mathrm{a}}(2)$ (see Fig. 12) are observed. This is the evidence of the existence of the intra-cluster magnetic order as shown also from the magnetization data below $300 \mathrm{~K}$ (see Fig. 7). Moreover, the change of the EPR intensity vs. $T$ is rather peculiar, consisting of an absolute maximum at $230 \mathrm{~K}$ and of a shoulder at higher temperatures between $280 \mathrm{~K}$ and $260 \mathrm{~K}$. 


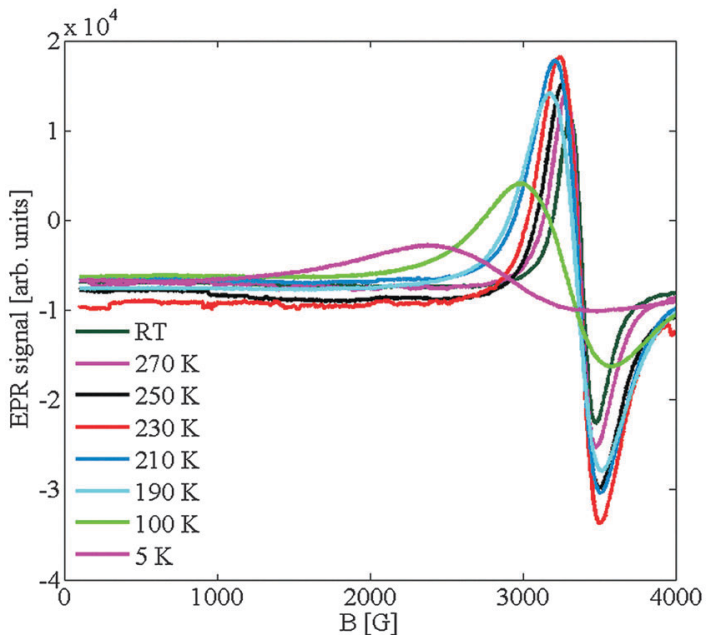

Fig. 14 EPR spectra recorded from $5 \mathrm{~K}$ to RT at selected temperatures.

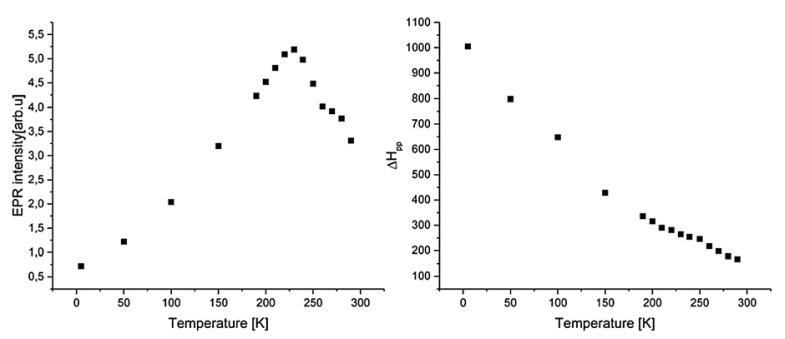

Fig. 15 EPR signal intensity vs. T (left) and EPR linewidth vs. T (right).

The latter occurs in the same temperature range where a plateau in the superparamagnetic-like normalized contributions to $t_{\mathrm{a}}(1)$ and $t_{\mathrm{a}}(2)$ (see Fig. 12) is observed. Therefore, this evidence can be interpreted to be due to the establishment of an inter-cluster magnetic order. Moreover, it has to be noted that $280 \mathrm{~K}$ and $260 \mathrm{~K}$ are close to those temperatures, which ref. 10 and 11 interpreted as ordering temperatures. By lowering $T$ below $260 \mathrm{~K}$, the EPR intensity increases further up to $230 \mathrm{~K}$. This is due to the fact that the long range coupled systems behave as superparamagnets, which means that in this temperature range the thermal energy is larger than the anisotropy barrier. At $230 \mathrm{~K}$ the system dynamics strongly depends on the height of the anisotropy barrier and it becomes gradually blocked by further decreasing $T$. Interestingly, at the same temperature the superparamagneticlike component of the Mössbauer spectra disappears and the system evolves into a "blocked" state.

\section{Discussion}

The structural characterization indicates the occurrence in $\mathrm{Pb}_{2} \mathrm{FeMoO}_{6}$ of a superstructure related to iron/molybdenum cation ordering, giving rise to a rock-salt distribution at the perovskite B site, with the presence of about $22 \%$ of antisite defects. Based on BVS calculations, the oxidation states of iron and molybdenum at room temperature are expected to be +3 and +5 , respectively, in good agreement with the nominal stoichiometry of the compound. On the other hand, the presence, indicated by Mössbauer spectroscopy, of two equally weighted iron sites characterized by slightly different oxidation states should correspond to iron ions presenting a different chemical environment. This scenario can be understood by considering a distribution of the antisite defects into small clusters, or better, nanometer-sized antiphase domains. ${ }^{26}$ Such behavior is reported also for the parent compound $\mathrm{Sr}_{2} \mathrm{FeMoO}_{6}$, where the formation of antiphase regions of average size ranging between 8 and $13 \AA$ is favored with respect to a random distribution of antisite defects or to the formation of large antiphase domains. Also in the present case this situation is considered most likely. In fact, the presence of antiphase domains with size exceeding the coherence length of $\mathrm{X}$-rays and neutrons would not contribute to the mixed occupancy at the Fe1 and Mo1 sites in the structural refinement of powder data. On the other hand, in order to fulfill simultaneously the conditions involving $22 \%$ of antisite defects (indicated by the structural characterization) and the presence of two different, equally weighted, iron populations (suggested by Mössbauer spectroscopy), the presence of nanometer-sized antiphase domains must be taken into account. The different iron oxidation states may originate from the site or the anti-site position of the metal ion or may be related to differences in the values for the Fe-Fe and Fe-Mo super-exchange integrals. If the iron oxidation is mainly due to the metal site localization, the ratio between the contributions of the two Mössbauer subspectra to the total thickness must be related to the antisite percentage. In the second case, the ratio is associated with the percentage of sites laying on the boundaries between site and anti-site regions that can also be greater than the antisite percentage, depending on the antiphase domain size. As a limited case, a single isolated ion in the antisite location will affect the electronic state of six ions. An average diameter of 16-20 A, corresponding to 4-5 simple perovskite cells, can be estimated by geometric modelling of the Fe/Mo distributions; only in this case indeed the number of iron ions involved in the antiphase boundaries (characterized by at least one $\mathrm{Fe}-\mathrm{O}-\mathrm{Fe}$ bond), and the amount of "ordered" iron ions (surrounded solely by molybdenum) would be almost equivalent. The magnetic properties of the compound result to be strongly dependent on the structural features of the system: a superparamagnetic-like evolution of the Mössbauer and EPR spectra indicates at RT the presence of weakly interacting regions of the sample, characterized by different anisotropy. Within the characteristic time scale of Mössbauer spectroscopy, part of them shows at RT static and saturated moments that become fully coupled to the rest of the sample below $250 \mathrm{~K}$, with a blocking temperature of $230 \mathrm{~K}$. On the other hand, a long range magnetic order is detected at $270 \mathrm{~K}$ by neutron diffraction and magnetometry, clearly indicating the ferrimagnetic nature of the ordered matrix. In addition, low-field magnetization measurements indicate a superparamagnetic-like blocking at $55 \mathrm{~K}$. This process, involving the resultant moments within the nanometer-sized antiphase domains, probably corresponds to the blocking observed by Mössbauer at $230 \mathrm{~K}$, by considering the different time scales of the two techniques. The superparamagnetic-like behavior is ascribed, in this case, to the thermal evolution of the anisotropy 
barriers, which result to be quite low as confirmed by the high values of the normalized superparamagnetic-like contributions to the Mössbauer spectra (see Fig. 12). The low magnetic anisotropy is in agreement with the pseudocubic character of the magnetic structure, where the easy axis of magnetization has the same probability to lie along one of the three [100] directions of the cubic nuclear cell and consequently the energy barrier separating the different solutions can be particularly small. Besides symmetry considerations, the presence of antiphase domains as well as charge delocalization may break the long range order constituted of superexchange mediated $\mathrm{Fe}^{3+}-\mathrm{O}-\mathrm{Mo}^{5+}$ interactions, determining the soft character of the compound. The magnetometric analyses give $\Theta_{\mathrm{CW}}=-55 \mathrm{~K}$, determined by a linear fit of the inverse magnetic susceptibility in the high- $T$ region $(T>400 \mathrm{~K})$, confirming the global AFM character of the superexchange interactions, in agreement with the ferrimagnetic structure indicated by neutron diffraction. The $\left|\theta / T_{\mathrm{N}}\right|$ ratio significantly lower than 1 should correspond to the presence of antiphase boundaries at the nanoscale together with Mo ion valence changes as a function of temperature yielded by charge delocalization mechanisms. ${ }^{11}$ However, if one considers $\mathrm{Pb}_{2} \mathrm{FeMoO}_{6}$ to be constituted of $\mathrm{Fe}^{3+}$ in a highspin electronic configuration and $\mathrm{Mo}^{5+}$ in equal parts, the expected number of effective Bohr magnetons for f.u. would be $6.17 \mu_{\mathrm{B}}$. The analysis of the inverse susceptibility in the paramagnetic region detected above room temperature gives $\mu_{\text {eff }}=(5.55 \pm 0.25)$ f.u. In the ordered region, the observed saturation magnetization is $\mathbf{1 . 7 5} \mu_{\mathrm{B}}$ per f.u., which is in good agreement with the value obtained on the basis of the magnetic structure by the difference of the moments refined for the two sites, hypothesizing at $5 \mathrm{~K}$ and after a field-cooling in $5 \mathrm{~T}$ the material to be virtually a monodomain. The neutron data indeed indicate the two independent magnetic sites to be ordered in an antiparallel way, as a consequence the resultant magnetization can be obtained as $\mu \mathrm{Fe} 1-\mu \mathrm{Mo} 1=3.06 \mu_{\mathrm{B}}-1.47$ $\mu_{\mathrm{B}}=1.59 \mu_{\mathrm{B}}$. It is noteworthy that the values observed for the two sites are not as expected for $\mathrm{Fe}^{3+}$ and $\mathrm{Mo}^{5+}$ (5 and $1 \mu_{\mathrm{B}}$ ), however the antisite fraction must be taken into account, whose contribution to the magnetic structure is to modify the observed magnetic moments at both the transition metal sites. In addition, the Mössbauer data indicate the atomic moment of iron to be $3.95 \mu_{\mathrm{B}}$, thus lower than expected, likely related to the presence of covalency phenomena or carrier delocalization. ${ }^{27}$ In some cases indeed, the Fe orbitals are indicated as contributing to the conduction, further lowering the localized moment. Hypothesizing that the TM in the antisite position orders according to the "host" site, the situation could be schematized as: site $\mathrm{Fe} 1=84 \% \mathrm{Fe} \uparrow, 16 \% \mathrm{Mo} \uparrow$; site $\mathrm{Mo} 1=73 \% \mathrm{Mo} \downarrow$, $27 \% \mathrm{Fe} \downarrow$. Within this framework, antiferromagnetic interactions would be expected for the Fe-O-Fe exchange paths located at the antiphase boundaries, which is in agreement with the Goodenough-Kanamori rule applied to undistorted iron(III)based perovskites. ${ }^{28,29}$ Using the atomic magnetization derived by Mössbauer spectroscopy for the iron ions, and considering the theoretical value of $1 \mu_{\mathrm{B}}$ for molybdenum in the $\mathrm{d}^{1}$ electronic configuration, the above mentioned model would imply, for the two sites: $0.83^{*} 3.95 \mu_{\mathrm{B}}+0.17^{*} 1 \mu_{\mathrm{B}}=3.45 \mu_{\mathrm{B}}$ (Fe1) and $0.73^{*} 1 \mu_{\mathrm{B}}+0.27^{*} 3.95 \mu_{\mathrm{B}}=1.80 \mu_{\mathrm{B}}$ (Mo1), which are in good agreement with the measured values of 3.06 and $1.47 \mu_{\mathrm{B}}$ obtained by neutron diffraction and, in general, the ones reported for other members of the $\mathrm{A}_{2} \mathrm{FeMoO}_{6}$ family. ${ }^{30}$ For both the sites, the neutron diffraction datum is underestimated, however the moment of iron and molybdenum could be reduced by the carrier delocalization observed in this system. ${ }^{11}$

\section{Conclusions}

$\mathrm{Pb}_{2} \mathrm{FeMoO}_{6}$ is a double perovskite characterized by rock-salt B-site cation ordering with the relevant presence of nanometersized antiphase domains. The lead atoms are statistically shifted off the centre of the oxygen coordination dodecahedron due to the "lone-pair" stereochemical effect of the $\mathrm{Pb}^{2+}$ ion. Mössbauer measurements show the presence of two iron sites, characterized by different values of the isomer shift and the hyperfine field, pointing to the presence of iron ions with different oxidation states (in the 0.3 electrons range) likely related to their inequivalent chemical surroundings at the antiphase boundaries and into the ordered matrix. The magnetic structure indicates the atomic moments of iron and molybdenum to be coupled in an antiparallel way, giving rise to a ferrimagnetic resultant with a characteristic Neél temperature of $270 \mathrm{~K}$. The SQUID and Mössbauer measurements are in agreement with the structural characterization, with the antiphase domains playing a central role in determining the macroscopic properties of the compound. Superparamagnetic-like behaviour is detected at RT on the timescale of Mössbauer spectroscopy, ascribed both to low anisotropy and to the weak interactions occurring between the iron ions at the antiphase boundaries and the ones belonging to the ordered matrix. By decreasing temperature, Mössbauer and EPR measurements indicate the increase of the magnetic correlations involving the two kinds of iron populations, and finally, below $230 \mathrm{~K}$, the superparamagnetic-like behaviour completely disappears and the hyperfine field distributions gradually evolve towards sharper shapes. Accordingly, neutron diffraction and magnetometry indicate long range correlations to take place below $270 \mathrm{~K}$, giving rise to a ferrimagnetic resultant calculated as the difference of the two independent magnetic sites: $\mu \mathrm{Fe} 1-\mu \mathrm{Mo} 1=3.06 \mu_{\mathrm{B}}-1.47 \mu_{\mathrm{B}}=1.59 \mu_{\mathrm{B}}$ per f.u. This value is in good agreement with the saturation magnetization $\mu_{\mathrm{S}}=1.75 \mu_{\mathrm{B}}$ displayed by the hysteresis loop, which in addition shows very small coercivity ascribed to low magnetic anisotropy.

\section{Acknowledgements}

F. O. thanks Fondazione Cariparma for financial support. G. S. and M. L. acknowledge "Ente Cassa di Risparmio di Firenze" for its financial support (Grant no. 2013.0364). F. M. and D. D. thank César De Juliàn Fernàndez for fruitful discussion. Corrado Rizzoli is acknowledged for his contribution in cif file preparation. 


\section{Notes and references}

1 Y. Shimakawa, M. Azuma and N. Ichikawa, Materials, 2011, 4, 153.

2 A. Gupta and J. Z. Sun, J. Magn. Magn. Mater., 1999, 200, 24.

3 K.-I. Kobayashi, T. Kimura, H. Sawada, K. Terakura and Y. Tokura, Nature, 1998, 395, 677.

4 A. Grimaud, K. J. May, C. E. Carlton, Y. L. Yueh-Lin Lee, M. Risch, W. T. Hong, J. Zhou and Y. Shao-Horn, Nat. Commun., 2013, 4, 2439.

5 Y. Tomioka, T. Okuda, Y. Okimoto, R. Kumai, K.-I. Kobayashi and Y. Tokura, Phys. Rev. B: Condens. Matter Mater. Phys., 2000, 61, 422.

6 R. P. Panguluri, S. Xu, Y. Moritomo, I. V. Solovyev and B. Nadgorny, Appl. Phys. Lett., 2009, 94, 012501.

7 D. Sánchez, J. A. Alonso, M. García-Hernández, M. J. MartínezLope, J. L. Martínez and A. Mellergård, Phys. Rev. B: Condens. Matter Mater. Phys., 2002, 65, 104426.

8 V. N. Singh and P. Majumdar, Europhys. Lett., 2011, 94, 47004.

9 M. Retuerto, M. J. Martínez-Lope, M. García-Hernández and J. A. Alonso, J. Phys.: Condens. Matter, 2009, 21, 186003.

10 Y. Zhang and V. Ji, J. Phys. Chem. Solids, 2012, 73, 1116.

11 Y. P. Liu, H. R. Fuh and Y. K. Wang, J. Phys. Chem. C, 2012, 116, 18032.

12 I. O. Troyanchuk, L. S. Lobanovsky, H. Szymczak and K. J. Bärner, J. Magn. Magn. Mater., 2000, 219, 163.

13 X. Yuan, M. Xu and Y. Chen, Appl. Phys. Lett., 2013, 103, 052411.

14 A. P. Hammersley, FIT2D: An Introduction and Overview, ESRF Internal Report, ESRF97HA02T, 1997.

15 L. C. Chapon, P. Manuel, P. G. Radaelli, C. Benson, L. Perott, S. Ansell, N. J. Rhodes, D. Raspino, D. Duxbury, E. Spill and J. Norris, Neutron News, 2011, 22, 22.
16 A. C. Larson and R. B. Von Dreele, General Structure Analysis System (GSAS), 2000; Los Alamos National Laboratory Report LAUR 86, 748.

17 H. B. Toby, J. Appl. Crystallogr., 2001, 34, 210.

18 B. J. Campbell, H. T. Stokes, D. E. Tanner and D. M. Hatch, J. Appl. Crystallogr., 2006, 39, 607.

19 G. Spina and M. Lantieri, Nucl. Instrum. Methods Phys. Res., Sect. B, 2014, 318, 253.

20 D. Altermatt and I. D. Brown, Acta Crystallogr., Sect. B: Struct. Sci., 1985, 41, 244.

21 K. Momma and F. Izumi, J. Appl. Crystallogr., 2011, 44, 1272.

22 F. Bødker, M. F. Hansen, C. B. Koch, K. Lefmann and S. Mørup, Phys. Rev. B: Condens. Matter Mater. Phys., 2000, 61, 6826.

23 M. A. Chuev, Dokl. Phys., 2012, 57, 421.

24 G. Long and F. Grandjean, Mössbauer Spectroscopy Applied to Magnetism and Materials Science, Plenum Press, New York, 1996, vol. 2 .

25 D. Delmonte, F. Mezzadri, C. Pernechele, G. Calestani, G. Spina, M. Lantieri, M. Solzi, R. Cabassi, F. Bolzoni, A. Migliori, C. Ritter and E. Gilioli, Phys. Rev. B: Condens. Matter Mater. Phys., 2013, 88, 014431.

26 C. Meneghini, S. Ray, F. Liscio, F. Bardelli, S. Mobilio and D. D. Sarma, Phys. Rev. Lett., 2009, 103, 046403.

27 D. D. Sarma, P. Mahadevan, T. Saha-Dasgupta, S. Ray and A. Kumar, Phys. Rev. Lett., 2000, 85, 2549.

28 J. B. Goodenough, J. Phys. Chem. Solids, 1958, 6, 287.

29 J. Kanamori, J. Phys. Chem. Solids, 1959, 10, 87.

30 C. Ritter, M. R. Ibarra, L. Morellon, J. Blasco, J. García and J. M. De Teresa, J. Phys.: Condens. Matter, 2000, 12, 8295. 